\title{
COVID-19 und seine Umwelt: Von einer Geschichte der Humanmedizin zu einer ökologischen Medizingeschichte?
}

\author{
Leander Diener
}

COVID-19 and Its Environment: From a History of Human Medicine Towards an Ecological History of Medicine?

\begin{abstract}
This paper is part of the Forum COVID-19: Perspectives in the Humanities and Social Sciences. The history of medicine is mostly written as a history of human medicine. COVID-19 and other zoonotic infectious diseases, however, demand a reconsideration of medical history in terms of ecology and the inclusion of non-human actors and diverse environments. This contribution discusses possible approaches for an ecological history of medicine which satisfies the needs of several current and overlapping crises.
\end{abstract}

Keywords: COVID-19, Ecology, Anthropocene, Cycles, Body Models

Dieser Beitrag ist Teil des Forums COVID-19: Perspektiven in den Geistes- und Sozialwissenschaften. Medizingeschichte wird gegenwärtig mit wenigen Ausnahmen als Geschichte der Humanmedizin geschrieben. COVID-19 und andere zoonotische Infektionskrankheiten legen allerdings nahe, Medizingeschichte grundsätzlich ökologischer zu denken und nicht-menschliche Akteur*innen sowie verschiedene „Umwelten“ miteinzubeziehen. Der vorliegende Beitrag diskutiert mögliche Ansätze für eine ökologische Medizingeschichte, die der überlagerung mehrerer Krisen gerecht wird und aktuelle Dringlichkeiten aufgreift.

Die Medizingeschichte basiert auf einer einfachen und meist unausgesprochenen Prämisse: Im Fokus des Interesses steht praktisch ausschließlich die Humanmedizin. ${ }^{1}$ Entsprechend sind die großen Themen der jüngeren Medizingeschichte - Sozialgeschichte der Medizin, Patient*innengeschichte, Körpergeschichte, Geschlechtergeschichte, Krankheitsgeschichte - in anthropomorphen Kontexten verortet. Nichtmenschliche Akteur*innen tauchen nur zuweilen als epistemische Objekte in einem bestimmten Experimentalsystem auf - beispielsweise im Fall von Louis Pasteur (Latour 1988) und Robert Koch (Gradmann 2005) oder in der Erforschung und Produktion von Wirkstoffen (Haller 2012). „Natürliche“ Umwelten werden entwe- 
der als externe Faktoren untersucht, zum Beispiel bezüglich spezifischer klimatischer Bedingungen (Sellers 2018) oder beim Studium von Überlebensstrategien prekärer Organismen als innere Milieus, die feindlichen externen Milieus gegenübergestellt werden (z.B. Geroulanos \& Meyers 2018; Heggie 2019). Die leitenden Körpermodelle sind entsprechend maschinelle Körper (Sarasin 2001; Rabinbach 1990), bakteriologische Körper (Berger 2009a) oder homöostatische Körper (Kury 2012; Jackson 2013): Körper also, die auf einer bestimmten Konzeption der Abgeschlossenheit von Organismen basieren. Diese Körpermodelle lassen sich schließlich mit Gewinn auf bestimmte politische Agenden hin untersuchen (für den bakteriologischen Körper siehe z.B. Sarasin et al. 2007).

Als sich im Zuge eines weit um sich greifenden Anthropological Turn in den 1970er und 1980er Jahren aufregende neue Perspektiven auf geisteswissenschaftliche Themen entwickelten, wurde auch die Medizingeschichte davon erfasst (Güttler 2019: 236). So wurde beispielsweise der traditionelle Anthropozentrismus (oder vielleicht eher: Androzentrismus) der althergebrachten Institution „Medizin“ mit feministischen und postkolonialen Ansätzen hinterfragt, etwa mit dem Argument, dass die moderne Medizin auf einem bestimmten problematischen männlichen, westlichen und weißen Standardkörper beruhe. Die gleichzeitige Ökologisierung des Theorieninventars in historischen Disziplinen, das heißt das Aufkommen von situierenden und lokalisierenden Herangehensweisen, hätte durchaus den tradierten, nun kritischen Standardkörper um das Attribut „human“ erweitern können. Warum dies nicht geschehen ist, soll jedoch nicht Gegenstand dieser kurzen Erörterung sein. Jedenfalls zog die Medizingeschichte anders als die Medizinanthropologie und auch die Wissenschaftsgeschichte nicht so bereitwillig mit, als es um die Integration der Natur und der Umwelt in den Untersuchungsbereich und um die entsprechende ökologische Re-Konzeptualisierung des Körpers und des Menschlichen ging: Die medizinische Umwelt wurde primär sozial gedacht. Und während sich die Medizinanthropologie und die Wissenschaftsgeschichte bereits seit Jahren mit dem diagnostizierten Zeitalter des Anthropozäns und den damit verbundenen Folgen beschäftigt, arbeitet sich die Medizingeschichte weiterhin an dem sich aus der Körpergeschichte der 1980er Jahre ergebenden Spannungsfeld zwischen essenzialistischem Biologismus und Sozialkonstruktivismus ab. ${ }^{2}$

Auf den ersten Blick scheinen Medizingeschichte und die Geschichte des Anthropozäns wenig miteinander zu tun zu haben; geht es hier um anscheinend relativ geschlossene gesellschaftliche Aushandlungsprozesse von Gesundheit und Krankheit, wird dort unter anderem das menschliche Eingreifen in die Erdgeschichte thematisiert. Wie genau die Veränderung der Umwelt medizinhistorisch aufgearbeitet werden kann und soll, 
ist eine offene Frage. Eine Zeitgeschichte der Medizin - und darauf läuft die Frage nach dem Anthropozän früher oder später hinaus - beschäftigt sich jedenfalls momentan eher mit anderen Faszinationsthemen: den Neurowissenschaften, hochtechnisierter Spitzenmedizin, der epidemiologischen Transition oder mit AIDS und anderen Infektionskrankheiten in einem Schnittbereich zur Medizinanthropologie. Die AIDS-Pandemie ist hier ein besonders aufschlussreiches Beispiel, zumal der HIV-Erreger relativ früh mit einer Form von Mensch-Tier-Übertragung in Verbindung gebracht wurde (Grmek 1990: 141-155). Ebenfalls war relativ früh klar, dass im Zentrum der Geschichtsschreibung von AIDS vor allem die soziale Konstruktion der Krankheit stand (Fee \& Fox 1992; McKay 2017). Diese historische Interpretation von AIDS war sowohl auf eine historiografische als auch eine soziopolitische Gewichtung der Zeit zurückzuführen und prägt die einschlägige Literatur bis in die Gegenwart.

Der Übergang ins 21. Jahrhundert brachte mit einer Häufung von epidemischen Infektionskranken wie SARS und Ebola schließlich aufs Tapet, wovor vonseiten der Tierseuchenüberwachung seit Jahrzehnten gewarnt worden war. Die maßlose Landnahme und damit einhergehende Verdrängung von Wildtieren sowie die Zunahme von Jagd und Konsumation provozierte eine Häufung sogenannter Zoonosen im ausgehenden 20. und frühen 21. Jahrhundert (Hahn et al. 2000: 613). Die COVID-19-Pandemie stellt lediglich das jüngste Beispiel dar, so kann man in verschiedenen Expertisen lesen; weitere zwischen Menschen und Tieren zirkulierende Viruserkrankungen werden mit großer Wahrscheinlichkeit folgen. Die drohende Gefahr von Zoonosen, aber auch die irreversible Realität von Antibiotikaresistenzen in der Landwirtschaft führte zum gesundheitspolitischen Projekt von One Health, in dem verschiedene internationale Organisationen dazu aufforderten, von der strikten Trennung in Human- und Veterinärmedizin abzusehen und der wechselseitigen Verflochtenheit der Gesundheit von Menschen und Tieren mehr Beachtung zu schenken. Das Wellcome-Trust-finanzierte historische Projekt zu One Health von Abigail Woods, Michael Bresalier, Angela Cassidy und Rachel Mason Dentinger untersuchte vor diesem Hintergrund historische Zusammenhänge, in denen Tier und Mensch enggeführt wurden (Woods et al. 2018). Abgesehen von diesem dezidiert auf die Schnittmenge von Human- und Veterinärmedizin fokussierenden Projekt widmeten sich weitere historische Projekte den Kreisläufen, die Mensch und Tier verbinden. Beispielhaft ist etwa Hannah Landeckers Arbeit über den Metabolismus von nicht-menschlichen und menschlichen Akteur*innen, in dem Abfallprodukte aus der industriellen tierischen Nahrungsmittelproduktion zirkulieren (Landecker 2019). 
Dass die Jahre 2020 und 2021 im Zeichen der COVID-19-Pandemie standen und stehen, lässt die Ausrichtung einer Medizingeschichte hinterfragen, in der Tiere höchstens peripher interessieren. Welche Agenda wird verfolgt, wenn Tierkörper und damit auch die natürliche Umwelt größtenteils ausgeklammert werden - oder vielmehr, wie müsste eine Agenda aussehen, die das nicht tut? Und wichtiger: Kann man sich das überhaupt noch leisten? Mit dieser zweiten Frage hängt eine weitere größere Aufgabe zusammen, die sich die Medizingeschichte stellen müsste: Wie findet die Medizingeschichte Anschluss an einige der brennendsten Themen des 21. Jahrhunderts, zum Beispiel die grundlegende Umgestaltung der Umwelt und die Klimaerwärmung? Eine Geschichtsschreibung, die einer gewissen Orientierungsleistung in der Gegenwart verschrieben ist, leistet im besten Fall einen Beitrag zur Diskussion von aktuellen Fragen anhand historischer Zusammenhänge. COVID-19 als Zoonose gibt den Blick frei auf eine globalisierte Menschheit, die nicht nur sozial auf der Welt, sondern auch biologisch mit der Welt verknüpft ist. Diese Interdependenzen können Angst machen, aber auch Grund zur Hoffnung geben - Hoffnung darauf, dass die Position des Menschen in der Welt wieder verhandelbar wird, was beispielsweise angesichts der Machtstellung neoliberaler Institutionen und einer verfestigten Ressourcenlogik im 21. Jahrhundert kaum mehr für möglich gehalten wurde. Das ökonomische Wachstumsparadigma und die Idee des freien Marktes stellten im Sommer 2020 nicht nur die Rolle des Staates gegenüber einem überforderten Gesundheitswesen zur Debatte. Infrage gestellt wurde auch die Idee, dass sich menschliche Körper dank einem pharmaceutical-technological fix gegen alle möglichen Gefahren aus der Umwelt absichern können.

Diese Situation fordert gewissermaßen eine Neuverhandlung alter Gewissheiten in der Medizingeschichte. Wie könnte also eine ökologisch gedachte Medizingeschichte aussehen? Julie Livingston schlug ganz grundsätzlich vor, Medizingeschichte als „Vorgeschichte“ unserer neuen „Normalität der klimawandel-induzierten Ausnahmesituation“ neu zu denken (Langstaff 2020). COVID-19 erscheint gemäss Richard C. Keller lediglich als „Facette einer breiteren Erfahrung der spätkapitalistischen Moderne, oder des gegenwärtigen Anthropozän-Höhepunkts“ (Langstaff 2020). In diesem Sinne könnte zunächst empirisch nach den Auswirkungen historischer Formationen des Gesundheitswesens auf die Umwelt gesucht werden, etwa in Form von materiellen Kreisläufen (z. B. pharmazeutische Produkte wie Hormone im Grundwasser) oder von Abfallprodukten aus dem Gesundheitswesen (z. B. infektiöser Abfall). Dann könnte nach den gesellschaftlichen, kulturellen, politischen und institutionellen Bedingungen für diese Formationen gefragt werden. Anschließend müssten die Mechanismen und Infrastrukturen untersucht werden, die dafür verantwortlich wa- 
ren, dass das Wissen über derartige Kreisläufe und ökologische Vernetzungen oft prekär war und zuweilen unterdrückt wurde. Solche Formen von Wissen beziehungsweise Nicht-Wissen lassen gesellschaftliche Reaktionen auf COVID-19, den Klimawandel und andere Problemlagen vergleichbar werden.

Laura J. Martin, Assistenzprofessorin für Environmental Studies, wies bereits im April 2020 darauf hin, dass man über die Ähnlichkeiten und Unterschiede zwischen der COVID-Pandemie und der Klimaerwärmung nachdenken müsste (Martin 2020). Wie kommt es, dass in einem Fall unzählige (tendenziell jüngere) Menschen gewaltige Veränderungen in ihrem Alltag in Kauf nehmen, während im anderen Fall grundlegende Änderungen in Produktion und Konsum nur zögerlich umgesetzt werden? Wie soll man sich zur Verschränkung der beiden Krisen verhalten, etwa hinsichtlich des Backlashs im Klimaschutz, der durch immense Infrastrukturprogramme und gelockerte Umweltschutzbestimmungen zur Stabilisierung der nationalen Wirtschaft in China und in den USA ausgelöst wird?

Eine weitere Möglichkeit könnte darin liegen, die der Medizin zugrunde liegenden Körpermodelle zu untersuchen - von menschlichen und nichtmenschlichen Körpern. Medizinische Körpermodelle und damit zusammenhängende Körperbilder sind ein zentraler Aspekt von Subjektivierung und politischer Agency. Auf diese Weise käme man schnell auf tierethische und tierrechtliche Diskussionen, aber auch auf soziale und politische Fragen: Körperbilder erlauben einen tiefgreifenden Blick auf das Selbstverständnis und die Handlungsfähigkeit von politischen Subjekten, wie etwa Philipp Sarasin anhand der "reizbaren Maschine“ zeigt. ${ }^{3}$ Je nach politischem Projekt muss danach gefragt werden, auf welche Weise Körperbilder Entscheidungen beeinflussen. In diesen Formen des Selbstbezugs treten immer bestimmte Verhältnisse zu „natürlichen“ und „kulturellen“ Umwelten zutage. Körpermodelle wie der maschinelle, der bakteriologische oder der homöostatische Körper operieren beispielsweise in ungesunden, feindlichen Umwelten. In diesem Überlebenskampf ist der Spielraum für Interventionen in die Umwelt entsprechend groß. Hier überlagern sich also anthropologische Selbstbezüglichkeiten, Umweltverhältnisse, Wissensordnungen und Handlungslogiken in einer spezifischen Art und Weise. Ausgehend von COVID-19 als Zoonose wäre es daher für unsere Gegenwart naheliegend, Medizin und vor allem die verhandelten Körpermodelle im Zeichen einer kritischen ökologischen Medizingeschichte zu überdenken und sich von der strikten Trennung in Organismen (menschliche Körper) und deren Umwelten (nicht-menschliche Körper und „natürliche“ Umwelten) zu lösen beziehungsweise deren intrikate Verstrickungen in den Fokus $\mathrm{zu}$ rücken. 
Funding Open access funding provided by University of Zurich

Open Access Dieser Artikel wird unter der Creative Commons Namensnennung 4.0 International Lizenz veröffentlicht, welche die Nutzung, Vervielfältigung, Bearbeitung, Verbreitung und Wiedergabe in jeglichem Medium und Format erlaubt, sofern Sie den/die ursprünglichen Autor(en) und die Quelle ordnungsgemäß nennen, einen Link zur Creative Commons Lizenz beifügen und angeben, ob Änderungen vorgenommen wurden.

Die in diesem Artikel enthaltenen Bilder und sonstiges Drittmaterial unterliegen ebenfalls der genannten Creative Commons Lizenz, sofern sich aus der Abbildungslegende nichts anderes ergibt. Sofern das betreffende Material nicht unter der genannten Creative Commons Lizenz steht und die betreffende Handlung nicht nach gesetzlichen Vorschriften erlaubt ist, ist für die oben aufgeführten Weiterverwendungen des Materials die Einwilligung des jeweiligen Rechteinhabers einzuholen.

Weitere Details zur Lizenz entnehmen Sie bitte der Lizenzinformation auf http://creative commons.org/licenses/by/4.0/deed.de.

\section{Anmerkungen}

1 In einer COVID-19-Spezialausgabe der Zeitschrift Centaurus vom Mai 2020 behandeln nur zwei von insgesamt 15 Beiträgen die Rolle von Tier und Umwelt in der Pandemie (D’Abramo \& Neumeyer 2020; Green 2020). Gleiches gilt mit zwei Ausnahmen für die Sektion „Second Look: Pandemics“ der Zeitschrift Isis (Second Look: Pandemics 2020). Im ersten „Forum COVID-19“ der Zeitschrift NTM behandelt keiner von sechs Beiträgen die Rolle von Tier und Umwelt in der Pandemie. Im Gegensatz dazu widmet sich eine Ausgabe der Zeitschrift Environmental History dezidiert dem Gebot der Stunde, das Menschliche neu zu denken (Alagona et al. 2020). Während für die environmental history seit einiger Zeit Grundlagentexte vorliegen (Chakrabarty 2009; Ingold 2011), fehlt ein Text mit vergleichbarer Dringlichkeit für die Medizingeschichte.

2 Diese Charakterisierung der Medizingeschichte ist als heuristische Polemik zu verstehen und wird natürlich keineswegs allen Ansätzen gerecht. Zwischenstufen in Richtung einer ökologischen Medizingeschichte bilden Projekte, die den abgeschlossenen Körper historisieren und problematisieren. Dazu gehören beispielsweise Projekte, die sich mit Wirt-Parasit-Interaktionen in der Medizin (z. B. Berger 2009b; Méthot \& Dentinger 2016), mit Umweltkonzepten in den Lebenswissenschaften (z. B. Wessely \& Huber 2017; Benson 2020), mit interspezifischen Wissenstransfers (z. B. Kohler 1994; Creager 2001; Roelcke 2009), mit Antibiotikaresistenzen (z. B. Condrau 2009; Podolsky 2015; Gradmann 2016; Keck 2018), mit der industriellen Lebensmittelproduktion oder verschiedenen Formen von Krankheitsökologie (z. B. Anderson 2004; Honigsbaum 2016; Seth 2018; Lynteris 2019; Engelmann \& Lynteris 2020) auseinandersetzen. Ebenso aufschlussreich sind Arbeiten zur „Biomolekularisierung“ des Lebens (z. B. Kay 1993; Keller 1995; de Chadarevian 2002; Grote 2019; Morange 2020), die einen von mehreren wissenshistorischen Kontexten für den medizinhistorischen Anthropozentrismus bilden.

3 Mit der Agency von Tieren beschäftigt sich eingehend das interdisziplinäre Feld der Human-Animal Studies. Untersucht wird hier die Interaktion von Tieren und menschlichen Gesellschaften, häufig entweder als intime Beziehungen mit oder industrielle Nutzung von Tieren - beides Themen mit einer bestimmten politischen Agenda (Kompatscher 2019: 12-13). Aus der Perspektive nichtmenschlicher Akteure lassen sich demge- 
mäß soziale Ordnungen menschlicher Gesellschaften beleuchten (Roscher 2012). Einflussreich waren hier insbesondere Donna Haraway und Bruno Latour, indem sie die kulturell geprägten Grenzziehungen zwischen Tier und Mensch kritisch auf epistemische Hegemonien befragten (Latour 1995; Haraway 2007; Eitler \& Möhring 2008).

\section{Literatur}

Alagona, Peter et al. 2020. Reflections: Environmental History in the Era of COVID-19. Environmental History 25(4): 595-686.

Anderson, Warwick 2004. Natural Histories of Infectious Disease: Ecological Vision in Twentieth-Century Biomedical Science. Osiris 19(1): 39-61.

Benson, Etienne 2020. Surroundings: A History of Environments and Environmentalisms. Chicago: University of Chicago Press.

Berger, Silvia 2009a. Bakterien in Krieg und Frieden. Eine Geschichte der medizinischen Bakteriologie in Deutschland 1890-1933. Göttingen: Wallstein.

Berger, Silvia 2009b. Abschied vom Krieg? Latente Infektionen und neue biologische Modelle der Wirt-Parasit-Interaktionen in der Bakteriologie der Weimarer Republik. Infektion und Institution. Zur Wissenschaftsgeschichte des Robert Koch-Instituts im Nationalsozialismus. Hg. von Marion Hulverscheidt, Anja Laukötter. Göttingen: Wallstein: 17-41.

de Chadarevian, Soraya 2002. Designs for Life: Molecular Biology after World War II. Cambridge: Cambridge University Press.

Chakrabarty, Dipesh 2009. The Climate of History: Four Theses. Critical Inquiry 35(2): 197-222.

Condrau, Flurin 2009. Standardising Infection Control: Antibiotics and Hospital Governance in Britain, 1948-1960. Harmonizing Drugs: Standards in 20th Century Pharmaceutical History. Hg. von Christian Bonah, Christophe Masutti, Anne Rasmussen, Jonathan Simon. Paris: Éditions Glyphe: 327-339.

Creager, Angela N. H. 2001. The Life of a Virus: Tobacco Mosaic Virus as an Experimental Model, 1930-1965. Chicago: University of Chicago Press.

D'Abramo, Flavio und Sybille Neumeyer 2020. A historical and political epistemology of microbes. Centaurus. Spotlight Issue: Histories of epidemics in the time of COVID-19 62(2): 321-330.

Eitler, Pascal und Maren Möhring 2008. Eine Tiergeschichte der Moderne. Theoretische Perspektiven. Traverse. Zeitschrift für Geschichte 15(3): 91-106.

Engelmann, Lukas und Christos Lynteris 2020. Sulphuric Utopias. A History of Maritime Fumigation. Cambridge: MIT Press.

Fee, Elizabeth und Daniel M. Fox 1992. Introduction: The Contemporary Historiography of AIDS. AIDS. The Making of a Chronic Disease. Hg. von Elizabeth Fee, Daniel M. Fox. Berkeley/Los Angeles/Oxford: University of California Press: 1-19.

Geroulanos, Stefanos und Todd Meyers 2018. The Human Body in the Age of Catastrophe: Brittleness, Integration, Science, and the Great War. Chicago/London: The University of Chicago Press.

Gradmann, Christoph 2005. Robert Koch und die medizinische Bakteriologie. Göttingen: Wallstein.

Gradmann, Christoph 2016. Re-Inventing Infectious Disease: Antibiotic Resistance and Drug Development at the Bayer Company 1945-1980. Medical History 59(2): 155-180.

Green, Monica H. 2020. Emerging diseases, re-emerging histories. Centaurus. Spotlight Issue: Histories of epidemics in the time of COVID-19 62(2): 234-247.

Grmek, Mirko D. 1990. History of AIDS. Emergence and Origin of a Modern Pandemic. Übers. von Russel C. Maulitz und Jacalyn Duffin. Princeton: Princeton University Press.

Grote, Mathias 2019. Membranes to Molecular Machines. Active Matter and the Remaking of Life. Chicago: The University of Chicago Press.

Güttler, Nils 2019. „Hungry for Knowledge“: Towards a Meso-History of the Environmental Sciences. Berichte zur Wissenschaftsgeschichte 42(2/3): 235-258. 
Hahn, Beatrice H. und George M. Shaw, Kevin M. De Cock, Paul M. Sharp 2000. AIDS as a Zoonosis: Scientific and Public Health Implications. Science 287(5453): 607-614.

Haller, Lea 2012. Cortison. Geschichte eines Hormons, 1900-1955. Zürich: Chronos.

Haraway, Donna 2007. When Species Meet. Minneapolis: University of Minnesota Press.

Heggie, Vanessa 2019. Higher and Colder. A History of Extreme Physiology and Exploration. Chicago/London: The University of Chicago Press.

Honigsbaum, Mark 2016. „Tipping the Balance“: Karl Friedrich Meyer, Latent Infections, and the Birth of Ideas of Disease Ecology. Journal of the History of Biology 49(2): 261-309.

Ingold, Alice 2011. Écrire la nature. De l'histoire sociale à la question environnementale? Annales. Histoire, Sciences Sociales 66(1): 11-29.

Jackson, Mark 2013. The Age of Stress. Science and the Search for Stability. Oxford: Oxford University Press.

Kay, Lily E. 1993. The Molecular Vision of Life: Caltech, the Rockefeller Foundation, and the Rise of the New Biology. New York/Oxford: Oxford University Press.

Keck, Frédéric 2018. A Genealogy of Animal Diseases and Social Anthropology (1870-2000). Medical Anthropology Quarterly 33(1): 24-41.

Keller, Evelyn Fox 1995. Refiguring Life: Metaphors of Twentieth-Century Biology. New York: Columbia University Press.

Kohler, Robert E. 1994. Lords of the Fly: Drosophila Genetics and the Experimental Life. Chicago: University of Chicago Press.

Kompatscher, Gabriela 2019. Human-Animal Studies. Bridging the Lacuna between Academia and Society. Animals and their Relation to Gods, Humans and Things in the Ancient World. Hg. von Raija Mattila, Sanae Ito, Sebastian Fink. Wiesbaden: Springer VS: 11-22.

Kury, Patrick 2012. Der überforderte Mensch. Eine Wissensgeschichte vom Stress zum Burnout. Frankfurt am Main: Campus Verlag.

Landecker, Hannah 2019. A metabolic history of manufacturing waste: food commodities and their outsides. Food, Culture E Society. An International Journal of Multidisciplinary Research 22(5): 530-547.

Langstaff, Alex 2020. Pandemic Narratives and the Historian. Los Angeles Review of Books (May 18, 2020). URL: https://lareviewofbooks.org/article/pandemic-narratives-andthe-historian/ (1.1.2021).

Latour, Bruno 1988 [1984]. The Pasteurization of France. Übers. von Alan Sheridan und John Law. Cambridge, London: Harvard University Press.

Latour, Bruno 1995 [1991]. Wir sind nie modern gewesen. Versuch einer symmetrischen Anthropologie. Berlin: Akademie-Verlag.

Lynteris, Christos (Hg.) 2019. Framing Animals as Epidemic Villains. Histories of Non-Human Disease Vectors. Cham: Palgrave Macmillan.

Martin, Laura J. 2020. The Coronavirus and Climate Action. Observations (April 10, 2020). URL: https://blogs.scientificamerican.com/observations/the-coronavirus-andclimate-action/ (1.1.2021).

McKay, Richard A. 2017. Patient Zero and the Making of the AIDS Epidemic. Chicago: University of Chicago Press.

Méthot, Pierre-Olivier und Rachel Mason Dentinger 2016. Ecology and Infection: Studying Host-Parasite Interactions at the Interface of Biology and Medicine. Journal of the History of Biology 49(2): 231-240.

Morange, Michel 2020. The Black Box of Biology. A History of the Molecular Revolution. Übers. von Matthew Cobb. Cambridge/London: Harvard University Press.

Podolsky, Scott H. 2015. The Antibiotic Era: Reform, Resistance, and the Pursuit of a Rational Therapeutics. Baltimore: Johns Hopkins University Press.

Rabinbach, Anson 1990. The Human Motor. Energy, Fatigue and the Origins of Modernity. New York: University of California Press.

Roelcke, Volker 2009. Tiermodell und Menschenbild. Konfigurationen der epistemologischen und ethischen Mensch-Tier-Grenzziehung in der Humanmedizin zwischen 1880 und 1945. Kulturgeschichte des Menschenversuchs im 20. Jahrhundert. Hg. von Birgit Griesecke, Marcus Krause, Nicolas Pethes, Katja Sabisch. Frankfurt am Main: Suhrkamp Verlag: 16-47. 
Roscher, Mieke 2012. Human-Animal Studies. Version: 1.0. Docupedia-Zeitgeschichte (25.01.2012). URL: http://docupedia.de/zg/roscher_human-animal_studies_v1_de_ $2012(24.11 .2020)$.

Sarasin, Philipp 2001. Reizbare Maschinen. Eine Geschichte des Körpers 1765-1914. Frankfurt am Main: Suhrkamp Verlag.

Sarasin, Philipp, Silvia Berger, Marianne Hänseler und Myriam Spörri (Hg.) 2007. Bakteriologie und Moderne. Studien zur Biopolitik des Unsichtbaren 1870-1920. Frankfurt am Main: Suhrkamp Verlag.

Second Look: Pandemics 2020. Isis. A Journal of the History of Science Society (111): 781-837. Sellers, Christopher 2018. To Place or Not to Place: Toward an Environmental History of Modern Medicine. Bulletin of the History of Medicine 92(1): 1-45.

Seth, Suman 2018. Difference and Disease: Medicine, Race, and the Eighteenth-Century British Empire. Cambridge: Cambridge University Press.

Wessely, Christina und Florian Huber (Hg.) 2017. Milieu. Umgebungen des Lebendigen in der Moderne. Paderborn: Wilhelm Fink.

Woods, Abigail, Michael Bresalier, Angela Cassidy und Rachel Mason Dentinger 2018. Animals and the Shaping of Modern Medicine. One Health and its Histories. Cham: Palgrave Macmillan.

Hinweis des Verlags Der Verlag bleibt in Hinblick auf geografische Zuordnungen und Gebietsbezeichnungen in veröffentlichten Karten und Institutsadressen neutral.

\section{Leander Diener}

Institut für Biomedizinische Ethik und Medizingeschichte

Universität Zürich

Winterthurerstr. 30

8006 Zürich

Schweiz

leander.diener@uzh.ch 